\title{
Patient Dose Assessment During Radiological Examination: Case of the Vakinankaratra and Haute Matsiatra Regions of Madagascar
}

\author{
Vololoniaina Bernardine ${ }^{1,3}$, Said Soudjay ${ }^{1,2}$, Ramanandraibe Marie Jeanne ${ }^{3}$, Rabesiranana Naivo ${ }^{4}$, \\ Ralaivelo Mbolatiana Anjarasoa Luc ${ }^{1}$, Razakarimanana Tahiry ${ }^{1}$, Raharimboangy Veroniaina ${ }^{1}$ \\ ${ }^{1}$ Dosimetry and radiation protection, National Institute of Sciences and Nuclear Techniques (INSTN-Madagascar), Antananarivo, Madagascar \\ ${ }^{2}$ Department of Physics, Faculty of Sciences, University of Comoros, Moroni, Comores \\ ${ }^{3}$ Department of Physics, Faculty of Sciences, University of Fianarantsoa, Fianarantsoa, Madagascar \\ ${ }^{4}$ Department of Physics, Faculty of Sciences, University of Antananarivo, Antananarivo, Madagascar
}

Email address:

berimalala@gmail.com (V. Bernardine)

\section{To cite this article:}

Vololoniaina Bernardine, Said Soudjay, Ramanandraibe Marie Jeanne, Rabesiranana Naivo, Ralaivelo Mbolatiana Anjarasoa Luc, Razakarimanana Tahiry, Raharimboangy Veroniaina. Patient Dose Assessment During Radiological Examination: Case of the Vakinankaratra and Haute Matsiatra Regions of Madagascar. Radiation Science and Technology. Vol. 7, No. 2, 2021, pp. 21-26.

doi: $10.11648 /$ j.rst.20210702.11

Received: April 6, 2021; Accepted: April 24, 2021; Published: April 30, 2021

\begin{abstract}
The medical use of ionizing radiation is the largest and a growing man-made source of radiation exposure. The aim of this study is to assess the doses received by patients during radiological examinations in order to standardize the examination procedures and optimize the patient dose. Four most frequented hospitals, located in the Vakinankaratra and Haute Matsiatra regions of Madagascar, were investigated. Patients dose undergoing chest posterior-anterior (PA) and lateral (LAT), skull (PA, LAT), lumbar spine (PA, LAT), spine cervical (PA, LAT), abdomen (AP, LAT) and members (AP, PA, LAT) X-ray examinations were involved in this study. Entrance Skin Dose (ESD) was calculated using the X-ray radiation output and the exposure parameters (high voltage, tube loading, focus-patient distance). Thermoluminescent dosimeters were used to measure the X-rays radiation output. Conversion coefficients were used to relate ESD to the effective dose (ED). A total of 302 radiographic examinations were collected from the four hospitals during two months of 2019. The highest ESD (mGy) was found for the lumbar spine lateral projection, with an average value of $2.66 \mathrm{mGy}$. The highest value of ED was observed for the abdomen lateral projection with an average of $0.174 \mathrm{mSv}$. The ESDs and EDs reported in this study are generally lower than reference dose values published by the IAEA. This trend is an indication that the patient radiation protection practices in these four hospitals are already acceptable. The results of this study showed that there is a need to improve the radiodiagnostic procedures for reducing patient radiation dose without affecting the quality of the radiography image. The data of this work will be useful in contributing to the formulation of regional guidance levels.
\end{abstract}

Keywords: Patient Dose, Thermoluminescent Dosimeters, X-ray Radiography, Radiation Protection

\section{Introduction}

Over the past hundred years, X-rays have been used for diagnostic purposes. Diagnostic X-rays are used for identifying diseases and other issues during medical examinations. Indeed, X-ray diagnostics allow the exploration of internal anatomical structures by the image obtained following the patient's crossing by an X-ray beam.
However, radiation exposure during diagnostic X-ray examinations can cause at the same time harmful effects to the person undergoing exposure, if its use is not optimized. So, the radiation protection is very necessary. This is the reason why the medical use of ionizing radiation is subject to a significant regulatory control. In Madagascar, the use of the X-ray equipment as a medical diagnostic tool is growing. As the patient dosimetry study during the radiological examination is as a very important step for dose optimization [1, 2], this 
experimental study was performed to investigate the patient dose. Similar studies on Entrance Skin Dose (ESD) assessment had been already carried out in the Capital of Madagascar for pediatric patient undergoing chest X-ray examinations. Results demonstrated that the radiation dose levels for patients in radiodiagnostic require optimization process. [3] The objective of this study is to determine the ESD and Effective Dose (ED) for adult patients during radiographic X-ray examinations in the regions of Haute Matsiatra and Vakinankaratra.

\section{Materials and Method}

\subsection{Study Location}

Four hospitals were included in this work: two university hospitals H1 and H2 located in Fianarantsoa (Haute Matsiatra region) and two private hospitals $\mathrm{H} 3$ and $\mathrm{H} 4$ located in Antsirabe (Vakinankaratra region) as shown in figure 1. The criteria for the selection of the hospitals considered for this study is the high number of patients that visit the facility for $\mathrm{X}$ - ray examination.

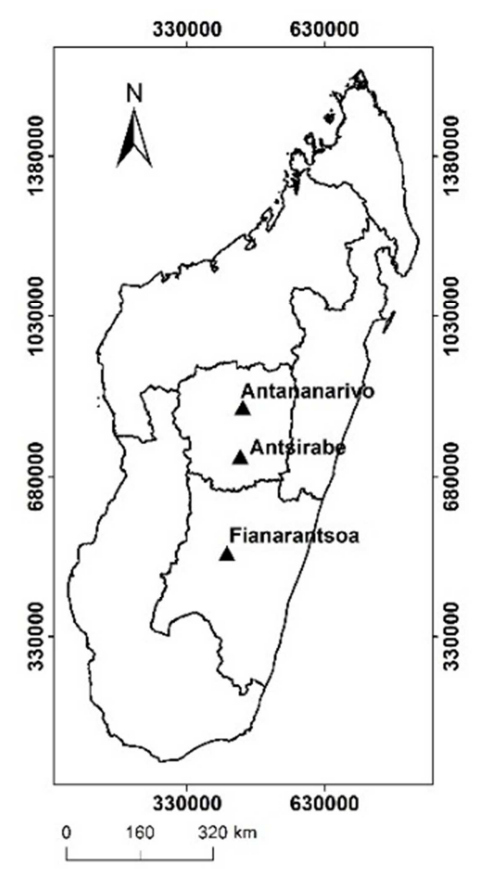

Figure 1. Geographic map of Madagascar.

\subsection{Patient Data Collection}

Radiation dose assessment was conducted for 302 patients during the period of study, from January to mars 2019. Inclusion criteria were the age of patients which were over 15 years and who underwent common radiographic examinations in the four selected hospitals.

\subsection{Data Collection}

Data collection was based on three categories:

1) The patient data as age, sex, weight and height;
2) The X-rays exposure parameters, that is high voltage, tube loading, Focus Film Distance (FFD), and patient thickness

3) Radiography equipment characteristic such as mark, model, etc.

\subsection{Thermoluminescents Dosimeters}

Dose values are needed to produce the X-ray output curve for each equipment. For this purpose, a thermoluminescent Dosimeter (TLD) was put on the radiographic table in the central beam axis. The focal spot to the detector distance (FDD) was set at $100 \mathrm{~cm}$. The setup of experimental study is illustrated in Figure 2.

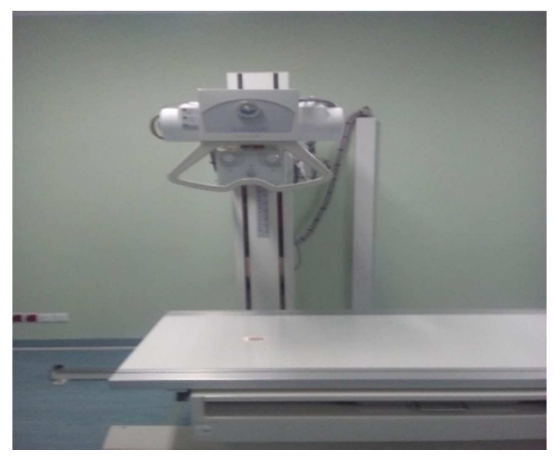

Figure 2. Radiographic device configuration for the X-ray radiation output measurement.

\subsection{Output X-rays Radiation Measurement}

The Focus Skin Distance (FSD) and exposure parameters $(\mathrm{kVp}$ and $\mathrm{mAs})$ used for selected examination were recorded for X-ray output calculation. The exposure versus high voltage variation according to the following equation

$$
\mathrm{OP}(\mathrm{mGy} / \mathrm{mAs})=\mathrm{f}(\mathrm{kV})
$$

has been plotted and the trend curve giving the ESD versus high voltage established according to previous studies $[4,5]$.

\subsection{Determination of Entrance Skin Dose}

In this study, the ESD for patients was assessed by an indirect method, using the empirical formula proposed by previous authors $[1,6,7]$, i.e.:

$$
\mathrm{ESD}=\mathrm{OP} * \mathrm{mAs} *\left(\frac{100}{F S D}\right)^{2} * \mathrm{BSF}
$$

with:

OP: X-ray output determined from the previous curve obtained by the formula (1)

mAs: tube loading

FSD: Focus Skin Distance

BSF: Backscatter factor.

Backscattering standard value, i.e. $\mathrm{BSF}=1.35$, was chosen according to [8].

\subsection{Determination of Effective Dose}

The effective dose (ED) is one of the parameters used to 
assess the relevance of examinations involving ionizing radiation. The ED value is obtained using the following formula [9]:

$$
\mathrm{E}=\mathrm{ESD} * C C_{E S D}
$$

where $C C_{E S D}$ is a conversion coefficient related to the radiographic $\mathrm{x}$-rays examination type, and provided by the NRPB-R262 report.

\section{Results and Discussions}

\subsection{Patient Characterization}

A total of 302 entries were collected from patients exposed to diagnostic X-rays examinations. Summary of all patient clinical parameters, for all examinations and hospitals, is displayed in Table 1.

Table 1. Patients characteristics for all hospitals.

\begin{tabular}{|c|c|c|c|c|c|}
\hline Examination & Projection & Number & Age Mean (min-max) & Weight Mean (min-max) & Height Mean(min-max) \\
\hline \multirow[t]{2}{*}{ Chest } & PA & 126 & $44(16-91)$ & $56(30-90)$ & $159(140-175)$ \\
\hline & LAT & 13 & $33(18-56)$ & $60(50-69)$ & $163(155-170)$ \\
\hline \multirow[t]{2}{*}{ Skull } & PA & 23 & $35(17-73)$ & $54(45-90)$ & $160(145-175)$ \\
\hline & LAT & 04 & $40(18-65)$ & $54(45-60)$ & $158(155-165)$ \\
\hline \multirow[t]{2}{*}{ Abdomen } & PA & 11 & $44(23-89)$ & $68(49-82)$ & $161(150-170)$ \\
\hline & LAT & 04 & $38(33-43)$ & $68(49-82)$ & $162(159-170)$ \\
\hline Lumbar spine & PA & 17 & $42(22-64)$ & $56(30-70)$ & $158(140-170)$ \\
\hline \multirow[t]{2}{*}{ Cervical spine } & PA & 07 & $39(16-59)$ & $64(50-90)$ & $159(144-166)$ \\
\hline & LAT & 06 & $38(16-59)$ & $65(50-90)$ & $159(144-166)$ \\
\hline Members & $\mathrm{AP} / \mathrm{PA}$ & 86 & $43(16-89)$ & $60(40-100)$ & $160(147-188)$ \\
\hline
\end{tabular}

The age of the patients ranges from 16 to 91 years old, while their weight and height range respectively from $30 \mathrm{~kg}$ to $100 \mathrm{~kg}$ and $140 \mathrm{~cm}$ to $188 \mathrm{~cm}$.

\subsection{Examination Frequency}

The different types of radiographic examination are shown in figure 3.

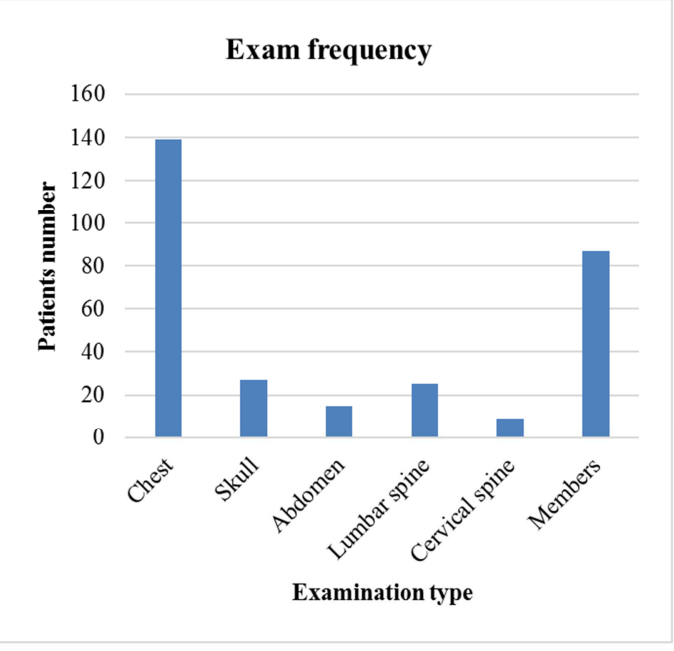

Figure 3. A bar chart showing the frequency of exam.

The figure 3 shows that chest and member examinations are the most frequent. The reasons are that most industries require that this test be performed for each new employee before starting work. In addition, it is also a rapid technique of medical imaging to detect heart-lung disease.

\subsection{Exposition Parameters}

The average, minimum and maximum values of tube potential $(\mathrm{kVp})$, tube loading (mAs) and focus - film distance
(FFD), for all examinations were recorded and are shown in table 2 .

Large fluctuation of the examination parameters has been established. It can be explained by the difference in the examination protocols adopted by each hospital and the patient size. According to previous studies made by Zahra Jomehzadeh et al., Rasuli et al and Guiswe Gnowe, the patient age is a significant parameter in the selection of the technical parameters and in considering the interpretation of radiological images. [10-12]. The choice of the high voltage, the tube loading and the FFD in this study was done for obtaining good quality according to the different morphology of the patients.

Table 2. Tables may span across both columns.

\begin{tabular}{lllll}
\hline Examination & Projection & $\begin{array}{l}\text { KVp Mean } \\
(\text { min-max) }\end{array}$ & $\begin{array}{l}\text { mAs Mean } \\
(\text { min-max })\end{array}$ & $\begin{array}{l}\text { FFD Mean } \\
\text { (min-max) }\end{array}$ \\
\hline Chest & PA & $74(48-105)$ & $19(5,4-63)$ & $154(100-214)$ \\
& LAT & $76(60-88)$ & $17(4,6-32)$ & $176(100-210)$ \\
Skull & PA & $71(49-90)$ & $17(8-63)$ & $108(96-130)$ \\
& LAT & $67(48-76)$ & $25(8-63)$ & $104(100-130)$ \\
Abdomen & PA & $70(53-78)$ & $23(8-63)$ & $111(100-123)$ \\
& LAT & $75(70-77)$ & $18,5(10-32)$ & $111(107-115)$ \\
Lumbar spine & PA & $69(50-86)$ & $22(3,2-50)$ & $120(98-180)$ \\
& LAT & $67(50-86)$ & $32(6,4-64)$ & $104(100-114)$ \\
Cervical & PA & $69(64-78)$ & $33(4,6-63)$ & $114(97-180)$ \\
spine & LAT & $70(64-78)$ & $32(4,6-63)$ & $107(97-137)$ \\
& AP/PA & $57(44-80)$ & $17(1-63)$ & $107(75-180)$ \\
\hline
\end{tabular}

\subsection{Radiation X-rays Output}

The tube loading was fixed at $20 \mathrm{mAs}$ and the tube high voltage values were set from $40 \mathrm{kVp}$ to $100 \mathrm{kVp}$. Changes in Kerma values are plotted and shown in figure 4. 


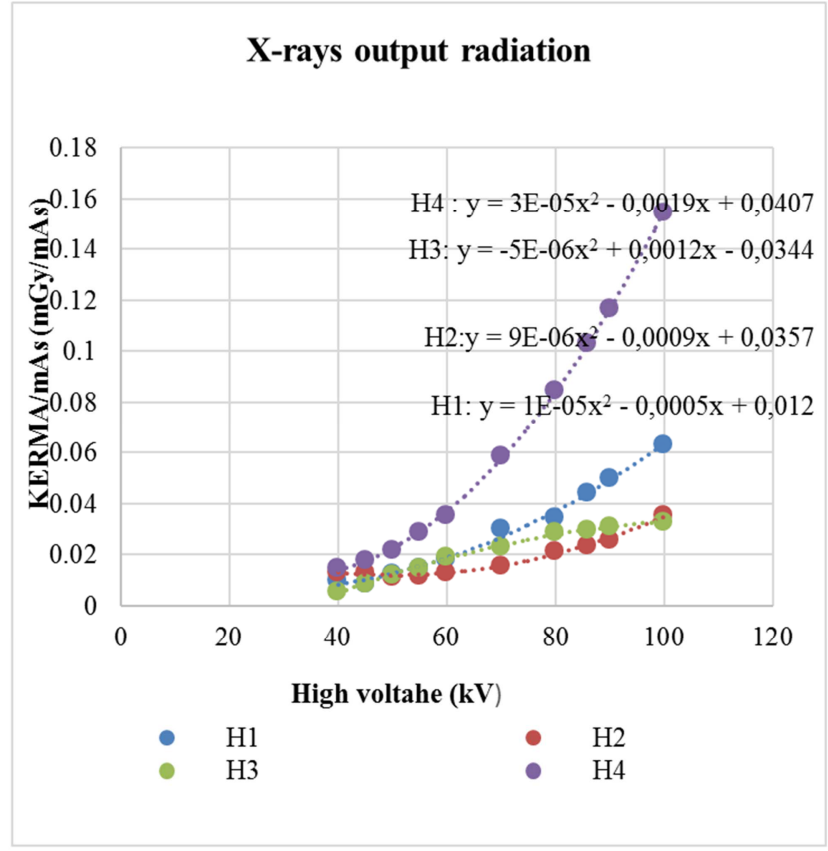

Figure 4. The radiation X-rays output for each radiography equipment.

Figure 4 shows that X-ray outputs are different for each $\mathrm{X}$-ray equipment unit. It depends on the radiographic equipment type, model and filtration. The obtained curves will be used to determine the output related to a specific high voltage.

\subsection{Entrance Skin Dose}

The data were recorded for the four hospitals. The hospital $\mathrm{H} 2$ carried out all the six examinations with two projections, while hospitals $\mathrm{H} 1$ and $\mathrm{H} 3$ conducted only three examinations. One of the reasons is that $\mathrm{H} 1$ and $\mathrm{H} 3$ are relatively new, and their available medical services are still not well known by the public.

The average values of the entrance skin dose (ESD) by hospital and for each examination were calculated according to the formula 1and are shown in Table 3.

Table 3. The ESD for each examination by hospital.

\begin{tabular}{llllll}
\hline Examination & Projection & \multicolumn{4}{l}{ Mean of Entrance Skin Dose (mGy) } \\
\hline Hospital & & H1 & H2 & H3 & H4 \\
\hline Chest & PA & 0,18 & 0,37 & 0,78 & 0,45 \\
& LAT & - & 0,23 & - & - \\
Skull & PA & 1.07 & 0.78 & - & 0.66 \\
& LAT & - & 2.68 & 1.10 & 0.33 \\
Abdomen & PA & - & 0.77 & - & 1.24 \\
& LAT & - & 1.16 & - & - \\
Lumbar spine & PA & - & 0.27 & - & 1.42 \\
& LAT & - & 1.00 & - & 3.20 \\
Cervical spine & PA & - & 1.37 & - & - \\
& LAT & - & 1.49 & - & - \\
Members & AP/PA & 0.09 & 0.63 & 0.72 & 0.23 \\
\hline
\end{tabular}

It can be seen that for all projections used for different radiography equipment, there is a wide variability in the ESD values. The main reasons are the difference in the radiographic equipment models, the exposure parameters and the X-ray radiation output. The lowest patient dose for chest and member examination was found at the hospital H1. The radiographic equipment was installed in this hospital in 2013, which is the newest among the investigated machines, and the lowest exposure parameters, notably the tube loading were used.

Table 4. Average entrance skin dose values for all hospitals.

\begin{tabular}{llll}
\hline Examination & Projection & $\begin{array}{l}\text { Mean of ESD } \\
(\mathbf{m G y})\end{array}$ & $\begin{array}{l}\text { DRLs in BSS-115 } \\
(\mathbf{m G y})\end{array}$ \\
\hline Chest & PA & 0.44 & 0.40 \\
& LAT & 0.23 & 1.5 \\
Skull & PA & 0.83 & 5 \\
Abdomen & LAT & 1.70 & 3 \\
& AP & 0.85 & 10 \\
Lumbar spine & LAT & 1.16 & 10 \\
& PA & 0.81 & 4.07 \\
Cervical spine & LAT & 2.66 & 30 \\
& PA & 1.37 & 10 \\
Members & LAT & 1.49 & 7 \\
\hline
\end{tabular}

The obtained values were lower than the DRLs provided by the IAEA, except for thorax projection (PA). In the present study, large dose variations for the common radiographic examinations have been determined. It has been established that the lumbar spine examination represents the highest patient exposition among the six studied projections. This is due the high charge value and the short focus-film distance. However, this dose value is largely inferior to the international published and established reference dose levels [13]. It means that patient radiation protection practice in the investigated hospitals is already acceptable.

A recent study on dose optimization for adult patient undergoing four common X-rays examinations was carried out in Haute Matsiatra region of Madagascar. Results, confirmed a good practice for patient dosimetry in the diagnostic radiology services. [14]

\subsection{Effective Dose}

According to the formula 2, the average value of the effective dose (ED) by hospital and for each examination is shown in Table 5 .

Table 5. Average value of effective doses by hospital and for each examination.

\begin{tabular}{llllll}
\hline Examination & Projection & \multicolumn{4}{l}{ Mean of Effective Dose (mSv) } \\
\hline Hospital & & H1 & H2 & H3 & H4 \\
\hline Chest & PA & 0,018 & 0,037 & 0,078 & 0,045 \\
& LAT & - & 0,023 & - & - \\
Skull & PA & 0,008 & 0,010 & - & 0,006 \\
& LAT & - & 0,020 & 0,006 & 0,002 \\
Abdomen & PA & - & 0,108 & - & 0,174 \\
& LAT & - & 0,162 & - & - \\
Lumbar spine & PA & - & 0,030 & - & 0,161 \\
& LAT & - & 0,019 & - & 0,060 \\
Cervical spine & PA & - & 0,054 & - & - \\
& LAT & - & 0,008 & - & - \\
Members & AP/PA & 0,004 & 0,003 & 0,002 & 0,001 \\
\hline
\end{tabular}

The highest ED variations were observed in abdomen PA 
and LAT examinations (hospitals $\mathrm{H} 4$ and $\mathrm{H} 2$ respectively), and the lowest were found in member examinations. Indeed, the abdomen is composed of soft tissues which are more sensitive to ionizing radiation than the others, and presents a high stochastic risk. Wide variations of patient doses are noted between hospitals, which are related to the difference in irradiation parameters and the conversion factor.

The results are then summarized to show the effects of examination and projection (table 6).

Table 6. The average value of effective dose for all hospitals.

\begin{tabular}{llll}
\hline Examination & Projection & $\begin{array}{l}\text { Mean of ED } \\
(\mathbf{m S v})\end{array}$ & $\begin{array}{l}\text { Effective doses in } \\
\text { CIPR 103 (mSv) }\end{array}$ \\
\hline Chest & PA & 0.044 & 0.42 \\
\multirow{2}{*}{ Skull } & LAT & 0.023 & - \\
& PA & 0,008 & 0.14 \\
Abdomen & LAT & 0,012 & - \\
\multirow{2}{*}{ Lumbar spine } & AP & 0,120 & 1.82 \\
& LAT & 0,160 & - \\
Cervical spine & PA & 0,092 & 0.66 \\
& LAT & 0,049 & - \\
Members & PA & 0,050 & - \\
\hline
\end{tabular}

The high effective dose observed in abdomen AP could be attributed to high value of ESD obtained for the diagnostic examinations. But the ED values in the present research were almost below the international recommendation provided by the ICRP $103[15,16]$. However low, the effective dose values contribute largely to the risk estimation with respect to each type of examination.

\section{Conclusion}

This study assessed the Entrance Surface Dose and Effective Dose for patients undergoing six X-ray examinations in four selected hospitals in Haute Matsiatra and Vakinankaratra regions of Madagascar. Effective Dose determination was carried out as it is an effective approach of risk assessment to patient exposed to medical radiation. The results show that the Entrance Surface Dose and Effective Dose values obtained were respectively lower than the international references provided by BSS-115 and ICRP 103, excepted for the chest Entrance Surface Dose. [14, 15]. This implies that the radiation exposure risk for the patients undergoing radiographic examinations in the hospitals included in this study is relatively low. However, even with such low level, it is always necessary to take precaution, due to the stochastic nature of the X-rays radiation effects. Indeed, the low dose value could anyway causes stochastic risks.

From this study, it is proposed to reduce patient dose while maintaining image quality by using high voltage $(\mathrm{kVp})$ and low charge (mAs). The findings of this work indicate also the need of quality assurance program. Clear protocol to describe patient radiation exposure during radiation examination is also suggested to avoid repeating X-ray examination.

Finally, this study was carried out only in two among 22 regions in Madagascar. Actions are underway to extend measurements into nationwide level. For this purpose, determination of patient doses through some regional approach and at national level is needed for establishing national diagnostic reference level.

\section{Acknowledgements}

The authors would like to thank the hospitals and their staff that participated in this study for allowing access to X-ray machines to carry out measurements. They also thank the Radioprotection and Dosimetry department and INSTN-Madagascar for the invaluable technical and logistical supports, as well as with the data processing.

\section{References}

[1] Eric K Ofori1, William K Antwi, Diane N Scutt and Matt Eric K Ofori1, William K Antwi, Diane N Scutt and Matt Ward 3, "Patient Radiation Dose Assessment in Pelvic X-ray Examination in Ghana", OMICS Journal of Radiology, 2013, volume2, issue 8 .

[2] Nsikan U. Esen, Rachel. I. Obed, "Doses Received by Patients during Thorax X-Ray Examinations", Iranian Journal of Medical Physics, (2013), volume 9, p. 245-251.

[3] Razakarimanana Tahiry, Ralaivelo Mbolatiana Anjarasoa Luc, Randriamora Tiana Harimalala, Raoelina Andriambololona3, Ravelomanatsoa Dieudonné Solofonirina, Ralainirina Dina Randriantsizafy, "Doses Evaluation of Some Body Organs of Pediatric Patients Undergoing Chest X-ray Examination Using Thermoluminescent dosimeter", Radiation Science and Technology, Science Publishing Groupe, volume 3, 2017, p. 24-28.

[4] Said Soudjay. «Efficiency of Thermoluminescent Dosimeters for Patient Dose Assessment in Diagnostic Radiology.», Master of Nuclear Physics, Department of Physics, Faculty of Sciences, University of Antananarivo, 2007.

[5] Marie Jeanne Ramanandraibe, Tiana Randriamora, Mbolatiana Ralaivelo, Raoelina Andriambololona, "patient Doses Assessment for Conventional Radiography in Madagascar", The Pharmaceutical and Chemical Journal, (2015), volume 2, p. $1-5$.

[6] L. R. Owoade, Isa Sambo and S. A. Tijani, "Assessment of entrance surface air kerma in patients undergoing chest X-ray from conventional diagnostic radiology in Ogun State, Nigeria”, academic journal, (2015), volume 7 (6), p. 117-120.

[7] M. T. Taha, F. H. Al-Ghorabie, R. A. Kutbi, W. K. Saib, "Assessment of entrance skin doses for patients undergoing diagnostic X-ray examinations in King Abdullah Medical City, Makkah, KSA", Journal of Radiation Research and Applied Sciences, (2015), volume 8, p. 100-103.

[8] Nadia O Alatta, Wadah. M. Ali 1, Hussein A Hassan, Ikhlas A Hasan, Sanaa A A Ibrahim, Elkhair M Ajbna, Mohaned Abdalla Omer, Albin Babu M Wilson, Altahir Abdalmalik, "Patient Radiation Dose Assessment in Routine Pelvic X-Ray Examination in Selected Hospitals in Khartoum State", International Journal of Science and Research, (2016), volume 7, issue 5, p. 741-744. 
[9] Sadeka Sultana Rubai, Md Shakilur Rahman, Santunu Purohit, Md Kawchar Ahmed Patwary, AKM Moinul Haque Meaze and Mamun AA, "Measurements of Entrance Surface Dose and Effective Dose of Patients in Diagnostic Radiography", Biomedical Journal of Scientific \& Technical Research (2018), volume 12 , issue 1 .

[10] Zahra Jomehzadeh, Ali Jomehzadeh, Mohammad Bagher Tavakoli, "Quality Control Assessment of Radiology Devices in Kerman Province, Iran», Iranian Journal of Medical Physics", (2016), volume 13, p. 25-35.

[11] Behrouz RASULI, Raheleh TABARI JUYBARI, Meysam FOROUZI, Mohammad GHORBANI, "Patient dose measurement in common medical X-ray examinations and propose the first local dose reference levels to diagnostic radiology in Iran", Polish Journal of Medical Physics and Engineering (2017), volume 3, p. 67-71.

[12] Guiswe Gnowe, Fouda H. P. Ekobena, Amvene J. Mbo, and Guena M. Neossi, "Exposure Levels of Adult Patients during Radiographic Examinations: Sinuses and Coastal Grill Cases at the Ngaoundere Regional Hospital, Cameroon", Hindawi Radiology Research and Practice, volume 2019.
[13] International Atomic Energy Agency, International Basic Safety Standards for Protection against Ionizing Radiation and for the Safety of Radiation Sources, IAEA Safety Series $n^{\circ} 115$, Vienna, Austria, 1996.

[14] Said Soudjay, Vololoniaina Bernardine, Ramanandraibe Marie Jeanne, Ramanantsizehena Georgette Vololona, Raboanary Roland, Randriamora Tiana Harimalala, "Application of Radiation Protection of Patients in Medical Diagnostic Radiology (Case of Public Hospitals, Fianarantsoa District, Haute Matsiatra Region, Madagascar)", Nuclear Science, Science Publishing Groupe, volume 6, 2021, p. 1-4.

[15] Nnamdi N. Jibiri and Christopher J. Olowookere, "Evaluation of dose-area product of common radiographic examinations towards establishing a preliminary diagnostic reference levels (PDRLs) in Southwestern Nigeria", (2017), Journal of applied clinical medical physics, volume 17, p. 392-304.

[16] ICRP 103, The 2007 Recommendations of the International Commission on Radiological Protection. 